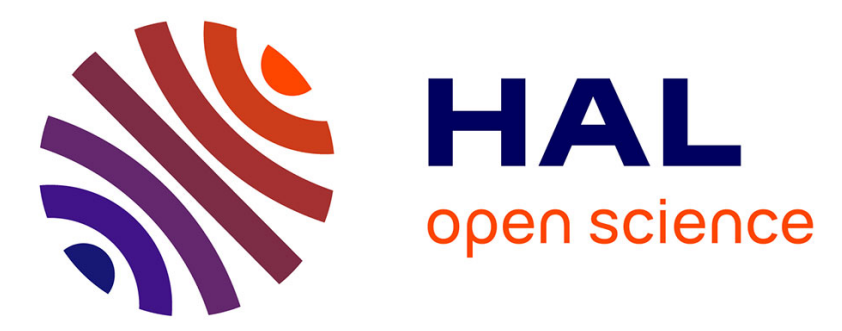

\title{
Defining scanning trajectory for on-machine inspection using a laser-plane scanner
}

Nguyen Duy Minh Phan, Yann Quinsat, Claire Lartigue

\section{To cite this version:}

Nguyen Duy Minh Phan, Yann Quinsat, Claire Lartigue. Defining scanning trajectory for on-machine inspection using a laser-plane scanner. JCM (International Joint Conference on Mechanics, Design Engineering and Advanced Manufacturing) 2018, Jun 2018, Cartagena, Spain. 10.1007/978-3-03012346-8_34.hal-01826484

\section{HAL Id: hal-01826484 https://hal.science/hal-01826484}

Submitted on 31 May 2019

HAL is a multi-disciplinary open access archive for the deposit and dissemination of scientific research documents, whether they are published or not. The documents may come from teaching and research institutions in France or abroad, or from public or private research centers.
L'archive ouverte pluridisciplinaire HAL, est destinée au dépôt et à la diffusion de documents scientifiques de niveau recherche, publiés ou non, émanant des établissements d'enseignement et de recherche français ou étrangers, des laboratoires publics ou privés. 


\title{
Defining scanning trajectory for on-machine inspection using a laser-plane scanner
}

\author{
Nguyen Duy Minh Phan · Yann Quinsat · Claire Lartigue \\ LURPA, ENS Paris-Saclay, Universite' Paris-Sud, Universite' Paris-Saclay, 94235 \\ Cachan, France
}

\begin{abstract}
Scan path planning for on-machine inspection in a 5-axis machine tool is still a challenge to measure part geometry in a minimum amount of time with a given scanning quality. Indeed, as the laser-plane scanner takes the place of the cutting tool, the time allocated to measurement must be reduced, but not at detrimental of the quality. In this direction, this paper proposes a method for scan path planning in a 5-axis machine tool with the control of scanning overlap. This method is an adaptation of a method dedicated to a robot that has proved its efficiency for part inspection.
\end{abstract}

Keywords: On-machine inspection; Laser-plane scanner; Overlap; 5-axis machine-tool, Digitization.

\section{Introduction}

Machining process monitoring is an essential challenge for the control of the quality of manufactured parts. To improve process productivity, part inspection procedures are increasingly integrated in the machining process as they allow high speed of inspection, measurement flexibility, and the possibility of $100 \%$ inspection [1]. Within this context, on-machine inspection becomes more and more popular as it allows a rapid decision-making with regards to part geometry conformity, and potential machining process corrections [2].

During on-machine inspection, the measurement of the part geometry is performed without removing the part from its set-up when the machining process is stopped. This facilitates the comparison of the machined part to its CAD model. Non-contact measuring techniques are generally used, as they represent a good compromise between data acquisition speed and resolution [3, 4]. As an example, a laser-plane sensor can be integrated in a milling machine tool by taking the place 
of the cutting tool to measure geometrical deviations of the machining part [2]. In this context, a challenging issue, still unsolved, concerns scanner path planning. Actually, scan path planning methods for part inspection in a 5-axis machine tool are little addressed in the literature. The main difficulty lies in the generation of a set of ordered scanner configurations (positions and orientations) defining the trajectory that answers classical scanning constraints, and is consistent with the description format of a tool trajectory for machining (Figure 1).

Classical scanning constraints, widely studied in the context of trajectory generation on Coordinate Measuring Machines, are related to visibility and quality criteria $[3,5,6,7,8]$. For on-machine inspection, the scanner trajectory is the succession of positions and orientations $\left(C_{E} ; V_{L} ; V_{C}\right)$. The driven point $C_{E}$ positions the scanning laser line in the field of view, and the couple of vectors $\left(\boldsymbol{V}_{\boldsymbol{L}} ; \boldsymbol{V}_{\boldsymbol{C}}\right)$ orients the scanner, with $\boldsymbol{V}_{\boldsymbol{C}}$ the director vector of the light-beam axis, and $V_{L}$, the director vector of the scanning line. As it takes the place of the tool, the scanner has thus a greater accessibility due to its possible movements: 5 degrees of freedom (dof) plus the spindle rotation for 5-axis machine-tool.

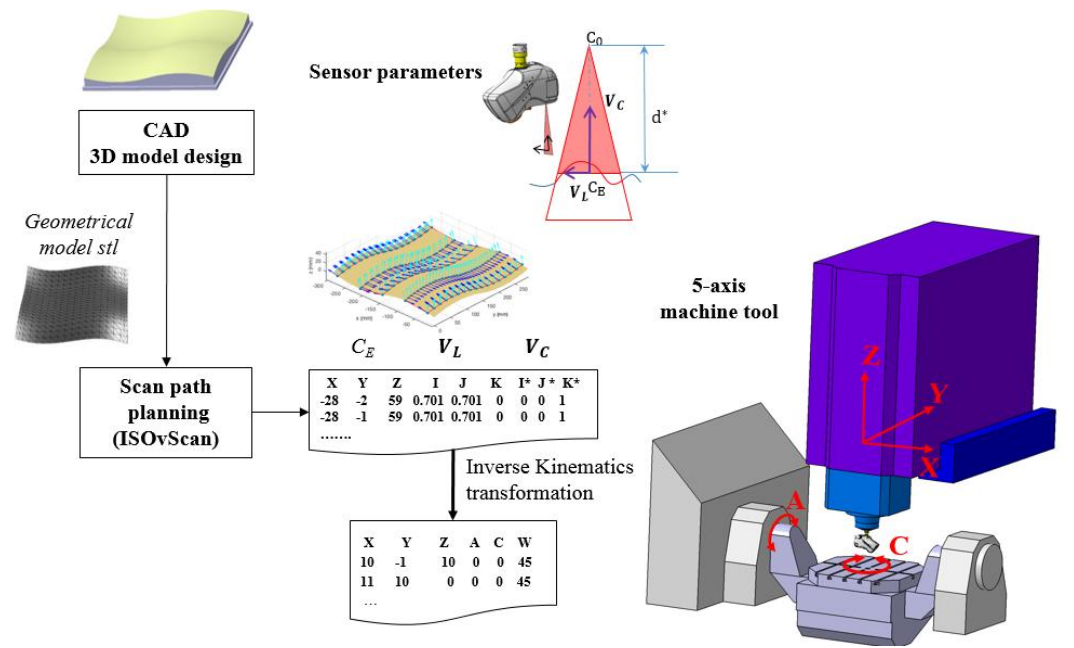

Fig.1. Parameters defining the scanner path for on-machine inspection

The path planning strategy consists in finding the trajectory of the driven point $C_{E}$, and the continuous evolution of the scanner orientations defined by the couple $\left(V_{L} ; V_{C}\right)$ allowing the scanning of the part. Although this problem has not yet been addressed in the literature, some methods dedicated to part inspection with industrial robots give some interesting insights [9, 10, 11, 12]. Most of these methods also rely on visibility and quality criteria.

This paper aims at defining a scanner trajectory well-adapted to on-machine inspection with 5-axis machine tool. In a previous work, we have developed a method that proved to be efficient for scanner path planning on industrial robots 
that integrates the control of overlap as an additional quality criterion [13]. This approach generates a scanning trajectory dedicated to a structure with 6 dof that must be adapted to 5-axis machine-tool. Indeed, the trajectory expressed in the part frame as a set of coordinates (X, Y, Z, I, J, K, I* $\left.\mathrm{J}^{*}, \mathrm{~K}^{*}\right)$ is expressed in the machine-tool frame thanks to the Inverse Kinematics Transformation (IKT). In the case of a RRTTT machine tool, the IKT leads to (X, Y, Z, A, C, W) in the articular space where $\mathrm{A}$ and $\mathrm{C}$ are the classical angles, and where $\mathrm{W}$ allows the spindle indexation. The main difficulty here is that the spindle indexation cannot be continuously controlled between two scanner configurations. It is thus necessary to fix the spindle indexation to a constant value $W_{c s t}$ for all the scanner configurations (Figure 1). The initial trajectory must thus be modified according to two different ways. Priority can be given to quality, with sensor orientation control, or priority is given to measuring time with overlap control.

This paper is organized as follows: the scan path planning method is presented briefly in Section 2 and is followed in section 3 by the method for adapting the trajectory to 5-axis scanning on a machine-tool. Finally, some conclusions are drawn in section 4

\section{Scan path planning method - ISOvScan}

The originality of the proposed method is the control of the scanning quality, while minimizing measuring time, based on the control of the scanning overlap. In a previous work, the importance of the overlap on the scanning quality has been discussed [13], leading us to develop the Iso-Overlap Scan path method (ISOvScan). This method is based on the stretching of the 3D mesh surface representative of the part to be measured on a $2 \mathrm{D}$ parametric surface by the LeastSquare Conformal Maps (LSCM) method [14]. The $\mathrm{n}$ triangular facets $T_{j}$ of the $3 \mathrm{D}$ surface are transformed into $\mathrm{n}$ facets $t_{j}$ in the $2 \mathrm{D}$ space. Then, equidistant paths, each one defined by a set of driven points, are generated in the $2 \mathrm{D}$ space, thus transformed in the 3D space by the inverse LSCM. For each driven point, the scanner orientations are finally calculated to satisfy quality constraints. The different steps of the method are briefly detailed in the next sections.

\subsection{Generation of the scanning driven points}

Driven points are defined in 2D as the intersection between parallel planes and the parametric surface. To control the overlap, parallel planes are equidistant of a value $I_{2 D}$, corresponding to the distance between two successive paths in the $2 \mathrm{D}$ space (Figure 2). As the objective is to control the overlap defined by the distance $I_{3 D}$ between two successive paths in the 3D space, a relation between $I_{2 D}$ and $I_{3 D}$ is established considering that the ratio of both values is equal to the proportionality 
coefficient of similar triangles $T_{j}$ and $t_{j}$ (Figure 2) where $A_{T j}$ and $a_{t j}$, are the area of the facet, respectively, $T_{j}$ and $t_{j}$.

Each driven point $c_{e i}$ is then transformed into its corresponding point $C_{E i}$ by the inverse transformation. Once all driven points are calculated, the next step is to determine sensor orientations for each driven point.

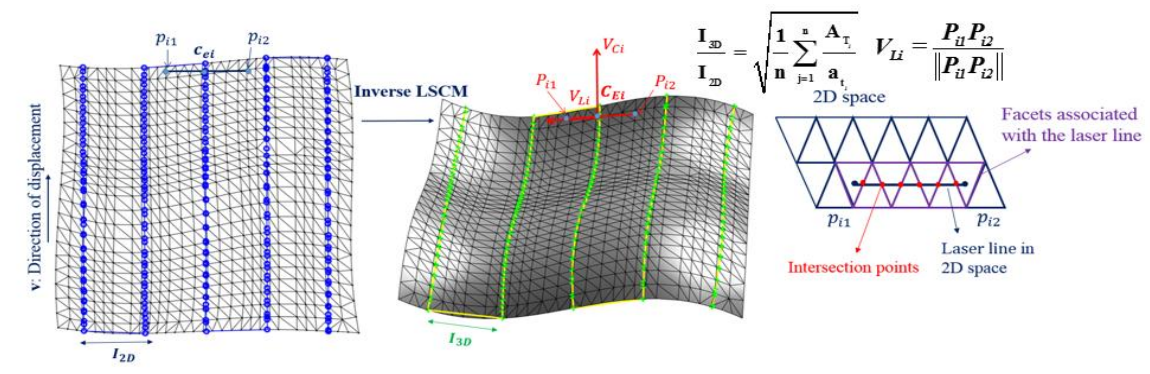

Fig.2. Calculation of the scan path

\subsection{Determination of scanner orientation}

Scanner orientations are determined in two steps: the vector director of the digitizing line $\boldsymbol{V}_{\boldsymbol{L}}$ is determined first, and then the light-beam axis $\boldsymbol{V}_{\boldsymbol{C}}$.

A constant scanning distance is first imposed for all the driven points to ensure that the measured surface at the driven point belongs to the Field Of View (FOV) of the scanner, and to also ensure an expected scanning quality, defined according to the scanner qualification [15].

The width of the laser line, $L_{\text {opt }}$ associated to this scanning distance is constant with respect to the scanned surface. In order to maximize the scanned surface, the laser line must be perpendicular to the direction of displacement along the scanner trajectory in the 3D space. The laser-line width $l_{\text {opt }}$ in the 2D space is defined from $L_{\text {opt }}$ and using the proportionality coefficient: $l_{\text {opt }}=L_{\text {opt }}\left(I_{3 D} / I_{2 D}\right)$. At each point $c_{e i}$, the laser-line is positioned perpendicularly to the path and centered at $c_{e i}$. The width $l_{o p t}$ defines the two end points $p_{i 1}$ and $p_{i 2}$ of the laser line (Figure 2). The coordinates of the corresponding points $P_{i 1}$ and $P_{i 2}$ are calculated using the LSCM inverse transformation. Thus, the director vector of the digitizing line $\boldsymbol{V}_{\boldsymbol{L} \boldsymbol{i}}$ at the driven point $C_{E i}$ is obtained from the coordinates of $P_{i 1}$ and $P_{i 2}$.

The vector of the light-beam axis $V_{C}$ is determined so that the scanning direction is always perpendicular to the surface. The local normal vector to the surface $\boldsymbol{n}_{C E i}$ at each driven point $C_{E i}$ is calculated as the mean value of all the normal vectors to the facets related to the laser line at $C_{E i}$. Finally, the director vector of the light-beam axis is defined at each driven point by: $\boldsymbol{V}_{\boldsymbol{C} i}=\boldsymbol{n}_{\boldsymbol{C E} \boldsymbol{i}}$. 


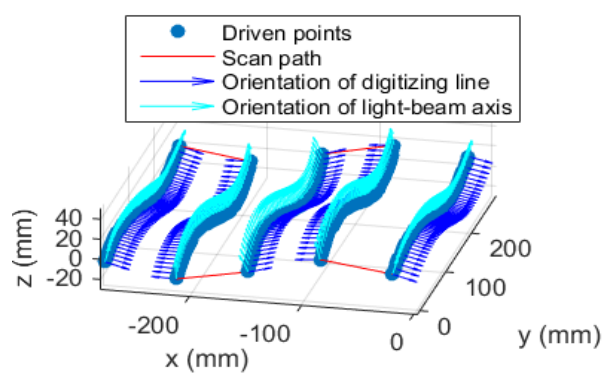

(a) Scan path generated by ISOvScan

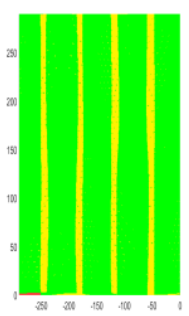

(b) Scanning simulation

Fig.3. Generated scan path and scanning simulation

The whole trajectory is thus obtained as a set of positions and orientations $\left(C_{E i}\right.$; $\left.V_{L i} ; V_{C i}\right)$. The scan path planning method with overlap control ISOvScan is implemented in Matlab $\odot$. This scan path is assessed by a simulator previously developed [16]. Green facets correspond to scanned facets, whereas yellow facets belong to overlap zones (Figure 3).

\section{Adaptation of the scanning trajectory to 5-axis machine-tools}

Before executing the measurement in the 5-axis machine tool (RRTTT architecture), the scanner configuration $\left(X_{p r}, Y_{p r}, Z_{p r}, I, \quad J, \quad K, I^{*}, J^{*}, K^{*}\right)$ corresponding to $\left(C_{E i} ; \boldsymbol{V}_{L i} ; \boldsymbol{V}_{C i}\right)$ and defined in the part frame must be transformed into the articular configuration $\left(X_{m}, Y_{m}, Z_{m}, A, C, W\right)$ in the machine frame. This transformation is carried out by IKT. Details of the geometrical modeling of the machine tool, and of the IKT are proposed in the appendix.

The values of the angles $\mathrm{A}$ and $\mathrm{C}$ are determined from the director vector of light-beam axis $V_{C}\left(I^{*}, J^{*}, K^{*}\right)$ in equation (1) where the matrix $\mathrm{M}$ is defined in appendix according to the kinematic transformation from the sensor frame to the part frame:

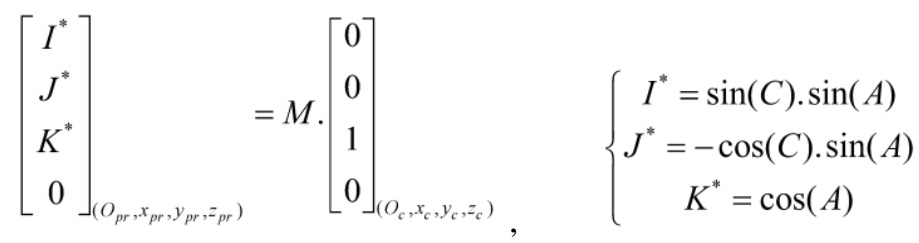

(1)

The value of angle $W$ is determined from $\boldsymbol{V}_{\boldsymbol{L}}(I, J, K)$ and the angles A, C: 
$\left[\begin{array}{c}I \\ J \\ K \\ 0\end{array}\right]_{\left(O_{p r}, x_{p r}, y_{p r}, z_{p r}\right)}=M\left[\begin{array}{l}1 \\ 0 \\ 0 \\ 0\end{array}\right]_{\left(O_{c}, x_{c}, y_{c}, z_{c}\right)}, \quad\left\{\begin{array}{c}I=\cos (C) \cdot \cos (W)-\cos (A) \cdot \sin (C) \cdot \sin (W) \\ J=\sin (C) \cdot \cos (W)+\cos (A) \cdot \cos (C) \cdot \sin (W) \\ K=\sin (A) \sin (W)\end{array}\right.$

As mentioned previously, the spindle indexation cannot be continuously controlled between two adjacent scanner configurations. The scanner trajectory must be transformed into $\left(X_{m}, Y_{m}, Z_{m}, A, C, W_{c s t}\right)$ where $W_{c s t}$ represents a constant value of the spindle indexation for all the scanner configurations. In a first approach, the value of $W_{c s t}$ is determined by calculating the angle between the mean value of all the director vectors of the digitizing line $\boldsymbol{V}_{\boldsymbol{L}}$ of the original scan path and the x-axis. The adaptation of the scan trajectory from ISOvScan to a trajectory well-adapted to a machine-tool is carried out in two ways. In the first method, the adapted scan path is computed with priority given to measuring time, with overlap control, and in the second method, priority is given to quality, with sensor orientation control.

In the first case, the adapted scan path respecting the control of overlap is obtained while keeping the director vector of the digitizing line $V_{L}$ from the original scan path, and by only transforming $\boldsymbol{V}_{\boldsymbol{C}}$ into the adapted vector $\boldsymbol{V}_{\boldsymbol{C}}$ '. Angles $\mathrm{A}$ and $\mathrm{C}$ are obtained from equation (2) with the original vector $\boldsymbol{V}_{L}$ and considering a constant value $W_{c s t}$. The adapted vector $\boldsymbol{V}_{C}$ ' is thus simply computed thanks to equation (1). Finally, the adapted scan path respecting the control of overlap defined by $\left(C_{E} ; \boldsymbol{V}_{\boldsymbol{L}} ; \boldsymbol{V}_{\boldsymbol{C}} \boldsymbol{\prime}\right)$ and the scanning simulation are shown in Figure 4

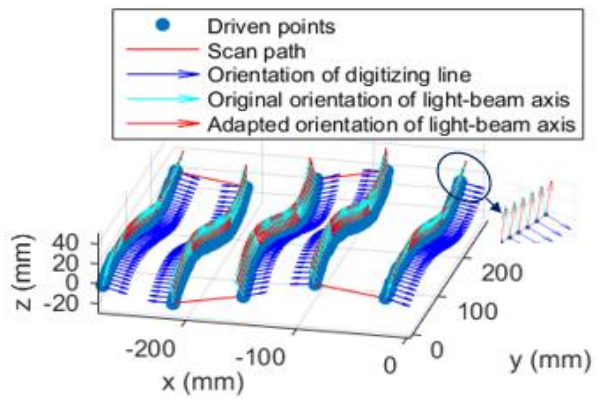

(a) Scan path with priority to the control of overlap

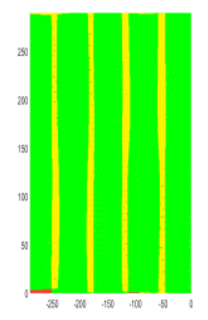

(b) Scanning simulation

Fig. 4. Scan path with priority to the overlap control and scanning simulation

In contrast, the adapted scan path with priority to scanning quality is carried out by keeping the director vector of the light-beam axis $\boldsymbol{V}_{C}$, and by calculating the adapted director vector of the digitizing line $V_{L}$ '. Angles $\mathrm{A}$ and $\mathrm{C}$ are calculated from the vector $\boldsymbol{V}_{\boldsymbol{C}}$ by equation (1). The adapted vector $\boldsymbol{V}_{\boldsymbol{L}}$, is then calculated by 
(2) with $W=W_{c s t}$. The scan path is also assessed by the simulator in Figure 5. The red color represents for the zones that are not scanned by the scanner.

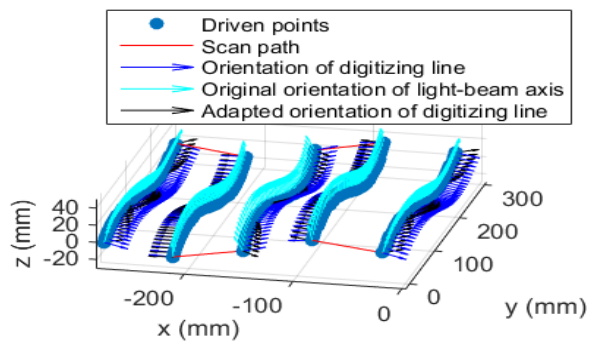

(a) Scan path with priority to the scanning quality

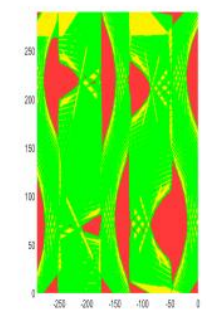

(b) Scanning simulation

Fig. 5. Scan path with priority to the scanning quality and scanning simulation

Results and discussion: The Results shown in Figures 4 and 5 bring out the good similarity between the original ISOvScan and the adapted scan path with priority to the control of overlap in terms of scanning quality and overlap zones. The computation of the angle deviation between $\boldsymbol{V}_{\boldsymbol{C}}$ and $\boldsymbol{V}_{\boldsymbol{C}}$ in Figure 4 has highlighted that this deviation is lesser than $22^{\circ}$. This ensures that the scanner view angle is still less than the maximum view angle $\left(60^{\circ}\right.$ for such a scanner [13]), which is linked to visibility and quality criteria. On the other hand, scanning simulation in Figure 5 (adapted scan path with priority to the scanning quality) presents many scanning holes (red color zones). Although the director vector of the light-beam axis $V_{C}$ is unchanged from the original scan path, the large deviations between the adapted director vector of digitizing line $\boldsymbol{V}_{\boldsymbol{L}}$ ' and $\boldsymbol{V}_{\boldsymbol{L}}$ result in many unscanned zones on the surface. This is likely due to the fact that $V_{L}$ is calculated from three parameters $\mathrm{A}, \mathrm{C}, \mathrm{W}$ while $\boldsymbol{V}_{\boldsymbol{C}}$ only depends on two variables $\mathrm{A}$ and $\mathrm{C}$.

In our case study, arbitrary choices of both the constant angle $W_{c s t}$ and the programming frame associated with the part setup may also influence results. Actually, the part setup is arbitrarily positioned in the machine working space without relation to scanning accessibility. However, these first results suggest that the adapted scan path with priority given to the control of overlap can be used as the suitable scan path for on-machine inspection in the 5-axis machine tool.

\section{Conclusion}

In this paper, we proposed a method to generate a laser-scanner trajectory suitable to 5-axis scanning in a machine tool. We successfully adapted a previous method that was developed for laser-scanning on a robot, which ensures scanning quality and the control of overlap between two adjacent scanning paths. As the kinematics of the machine-tool does not enable to directly apply the initial scan trajectory it must be adapted by fixing the value of the spindle indexation $W$. For this purpose, 
two different ways are explored: the value is fixed considering that priority is given to time measurement with overlap control, or priority is given to quality with sensor orientation control. First, results have demonstrated the relevancy of trajectory adaptation, with better results for the adapted scan path which prioritizes overlap control. Nevertheless, only a few experiments have been performed, and the work must be completed by investigating in particular the choice of the constant spindle indexation value, and the influence of the part setup on scanning performance. Future works, highly innovative and already in progress, concern the assessment of our method for on-machine measurement using a laser-plane in the 5 -axis machine tool.

\section{References}

[1] Chen, F.L., Su, C.T.: Vision-based automated inspection system in computer integrated manufacturing. The International Journal of Advanced Manufacturing Technology, 11(3):206-213, 1996.

[2] Quinsat, Y., Dubreuil, L., Lartigue, C.: A novel approach for in-situ detection of machining defects. The International Journal of Advanced Manufacturing Technology, 90(5-8):1625-1638, 2017.

[3] Lartigue, C., Quinsat, Y., Mehdi-Souzani, C., Zuquete-Guarato, A., Tabibian, S.: Voxel-based path planning for $3 \mathrm{~d}$ scanning of mechanical parts. Computer-Aided Design and Applications, 11(2):220-227, 2014.

[4] Poulhaon, F., Leygue, A., Rauch, M., Hascoet, J.Y., Chinesta. F.: Simulation-based adaptative toolpath generation in milling processes. International Journal of Machining and Machinability of Materials, 15(3-4):263-284, 2014.

[5] Bernard, A., Véron, M.: Visibility theory applied to automatic control of $3 \mathrm{~d}$ complex parts using plane laser sensors. CIRP Annals - Manufacturing Technology, 49(1):113 $-118,2000$.

[6] Prieto, F., Redarce, H.T, Lepage, R., Boulanger, P.: Range image accuracy improvement by acquisition planning. In Proceedings of the 12th conference on vision interface, Trois Rivieres, Québec, Canada, pages 18-21, 1999.

[7] Son, S., Park, H., Lee, K.H.: Automated laser scanning system for reverse engineering and inspection, International Journal of Machine Tools and Manufacture, vol. 42, no. 8, pp. 889-897, 2002.

[8] Mahmud, M., Joannic, D., Roy, M, Isheil, A., Fontaine, J.-F.: 3d part inspection path planning of a laser scanner with control on the uncertainty. Computer-Aided Design, vol. 43, no. 4, pp. 345-355, 2011.

[9] Wu, Q., Lu, J., Zou, W., Xu, D.: Path planning for surface inspection on a robot-based scanning system. In Mechatronics and Automation (ICMA), 2015 IEEE International Conference on, 2015, pp. 2284-2289.

[10] Koutecky, T., D. Palousek, D., and J. Brandejs, J.: Sensor planning system for fringe projection scanning of sheet metal parts. Measurement, vol. 94, pp. 60-70, 2016.

[11] Larsson, S., Kjellander, J. A. P.: Path planning for laser scanning with an industrial robot. Rob. Auton. Syst., vol. 56, no. 7, pp. 615-624, 2008.

[12] Mineo, C., Pierce, S. G, Nicholson, P.I., Cooper, I.: Robotic path planning for nondestructive testing--A custom MATLAB toolbox approach. Robot. Comput. Integr. Manuf., vol. 37, pp. 1-12, 2016. 
[13] Phan, N.D.M, Quinsat, Y., Lavernhe, S., Lartigue, C.: Path planning of a laserscanner with the control of overlap for 3D part inspection. In 11th CIRP Conference on Intelligent Computation in Manufacturing Engineering, CIRP ICME17, 2017.

[14] Bruno, L., Petitjean, S., Ray, N., Maillot, J.: Least squares conformal maps for automatic texture atlas generation. ACM Transactions on Graphics (TOG) 21(3):362-371, 2002.

[15] Mehdi-Souzani, C., Quinsat, Y., Lartigue, C., Bourdet, P.: A knowledge database of qualified digitizing systems for the selection of the best system according to the application. CIRP Journal of Manufacturing Science and Technology, 13:15-23, 2016.

[16] Phan, N.D.M., Quinsat, Y., and Lartigue, C.: Simulation of laser-sensor digitizing for on-machine part inspection. In Advances on Mechanics, Design Engineering and Manufacturing, pages 301-311. Springer, 2017.

[17] Lavernhe, S., Tournier. C, Lartigue, C.: Kinematic performances in 5-axis machining. Advances in Integrated Design and Manufacturing in Mechanical Engineering II, pages 489-503, 2007.

\section{Appendix : Modeling of the 5 axis milling machine structure}

The Mikron UCP 710 is a 5-axis milling center with an industrial numerical controller Siemens 840D. The architecture of this machine is CAXYZ, for which two rotations are applied to the part, and the tool orientation is fixed in the machine frame (Figure 6).

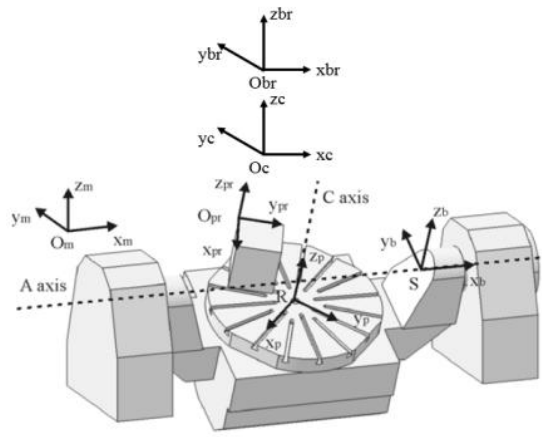

Fig. 6. Definition of different frames [17].

The different frames are defined from the architecture of the machine [17]:

- The spindle frame $\left(O_{b r}, x_{b r}, y_{b r}, z_{b r}\right)$ is linked to the spindle; the scanner frame $\left(O_{C}, x_{c}, y_{c}, z_{c}\right)$ is linked to the scanner,

- The machine frame $\left(O_{m}, x_{m}, y_{m}, z_{m}\right)$ is linked to the machine structure; its axes are parallel to the XYZ axes; $z_{\mathrm{m}}$ is parallel to the tool axis,

- The tilt frame $\left(S, x_{b}, y_{b}, z_{b}\right)$ is linked to the tilt table; $\mathrm{x}_{\mathrm{b}}$ is parallel to $\mathrm{x}_{\mathrm{m}}, \mathrm{S}$ is located on the A axis, 
- The table frame $\left(R, x_{p}, y_{p}, z_{p}\right)$ is linked to the rotary table; $\mathrm{z}_{\mathrm{p}}$ is parallel to $\mathrm{Z}_{\mathrm{b}}, \mathrm{R}$ is defined as the intersection between the $\mathrm{C}$ axis and the upper face of the table;

- The programming frame $\left(O_{p r}, x_{p r}, y_{p r}, z_{p r}\right)$ is linked to the part, which represents the frame used for scan path planning.

To transform between different frames, we define the matrix that converts a vector expressed in the one frame into another frame:

$$
\begin{aligned}
P_{c b r} & =\left[\begin{array}{cccc}
\cos (W) & \sin (W) & 0 & x_{\boldsymbol{o}_{c} \boldsymbol{o}_{b r}} \\
-\sin (W) & \cos (W) & 0 & y_{\boldsymbol{O}_{c} \boldsymbol{o}_{b r}} \\
0 & 0 & 1 & z_{\boldsymbol{O}_{c} \boldsymbol{o}_{b r}} \\
0 & 0 & 0 & 1
\end{array}\right] P_{b r m}=\left[\begin{array}{llll}
1 & 0 & 0 & x_{\boldsymbol{o}_{b r} \boldsymbol{o}_{m}} \\
0 & 1 & 0 & y_{\boldsymbol{o}_{b r} \boldsymbol{o}_{m}} \\
0 & 0 & 1 & z_{\boldsymbol{O}_{b r} \boldsymbol{o}_{m}} \\
0 & 0 & 0 & 1
\end{array}\right] ; \\
P_{m b} & =\left[\begin{array}{cccc}
1 & 0 & 0 & x_{\boldsymbol{o}_{m} s} \\
0 & \cos (A) & \sin (A) & y_{\boldsymbol{O}_{m} s} \\
0 & -\sin (A) & \cos (A) & z_{\boldsymbol{o}_{m} s} \\
0 & 0 & 0 & 1
\end{array}\right]
\end{aligned}
$$

where $P_{c b r}$ is the transformation matrix between the scanner frame and the spindle; $P_{b r m}$, the transformation matrix between the spindle frame and the machine frame and $P_{m b}$, the transformation matrix between the machine frame and the tilt frame.

$$
P_{b p}=\left[\begin{array}{cccc}
\cos (C) & \sin (C) & 0 & x_{S R} \\
-\sin (C) & \cos (C) & 0 & y_{S R} \\
0 & 0 & 1 & z_{S R} \\
0 & 0 & 0 & 1
\end{array}\right] ; P_{p p r}=\left[\begin{array}{cccc}
1 & 0 & 0 & x_{\boldsymbol{R} o_{p r}} \\
0 & 1 & 0 & y_{\boldsymbol{R} o_{p r}} \\
0 & 0 & 1 & z_{\boldsymbol{R} o_{p r}} \\
0 & 0 & 0 & 1
\end{array}\right]
$$

where $P_{b p}$ isthe transformation matrix between the tilt frame and the rotary table frame and $P_{p p r}$ is the transformation matrix between the rotary table frame and the part frame.

The kinematic transformation matrix $M$ from the sensor to the part is then defined as following: $\mathrm{M}=P_{p p r}^{-1} \cdot P_{b p}^{-1} \cdot P_{m b}^{-1} \cdot P_{b r m}^{-1} \cdot P_{c b r}{ }^{-1}$. 